\title{
Expression of STEAP1 and STEAP1B in prostate cell lines, and the putative regulation of STEAP1 by post-transcriptional and post-translational mechanisms
}

\author{
Inês M. Gomes ${ }^{1}$, Cecília R. Santos ${ }^{1}$, Cláudio J. Maia ${ }^{1}$ \\ ${ }^{1}$ CICS-UBI- Health Sciences Research Centre, University of Beira Interior, Av. Infante D. Henrique, Covilhã, Portugal \\ Correspondence to: Cláudio J Maia, email: cmaia@fcsaude.ubi.pt \\ Keywords: STEAP1, STEAP1B, Prostate cancer, Post-transcriptional, Post-translational \\ Received: April 10, $2014 \quad$ Accepted: June 10, $2014 \quad$ Published: June 10, 2014
}

This is an open-access article distributed under the terms of the Creative Commons Attribution License, which permits unrestricted use, distribution, and reproduction in any medium, provided the original author and source are credited.

\section{ABSTRACT}

STEAP1 gene is overexpressed in several kinds of tumors, particularly in prostate cancer. Besides STEAP1, there is another related gene, STEAP1B, which may encode two different transcripts. Although several studies have been pointing STEAP1 as a putative immunotherapeutic target and biomarker, the mechanisms underlying its regulation are not fully understood. In silico analysis allowed us to show that STEAP1 and STEAP1B share high homology, but with slight differences at structural level. Experiments with prostate cells showed that STEAP1B2 is overexpressed in cancer cells. Regarding STEAP1 regulation, it is demonstrated that the stability of mRNA and protein is higher in LNCaP than in PNT1A cells. Of note, serum triggered opposite effects in LNCaP and PNT1A in relation to STEAP1 stability, e.g., increasing it in PNT1A and decreasing in LNCaP. These results suggest that STEAP1 may be regulated by post-transcriptional and post-translational modifications (PTM), which may differ between non-neoplastic and neoplastic cells. These PTM are supported through in silico analysis, where several modifications such as $\mathbf{N}$-glycosylation, $\mathbf{N}$-Glycation, Phosphorylation and $\mathrm{O}$-linked $\boldsymbol{\beta}-\mathrm{N}$-acetylglucosamine, may occur in STEAP1 protein. In conclusion, these data indicate that STEAP1B2 is overexpressed in neoplastic cells, and PTM may be involved in regulation of STEAP1 expression in prostate cells.

\section{INTRODUCTION}

The six transmembrane epithelial antigen of the prostate 1 (STEAP1) is overexpressed in prostate cancer, and other malignant tumors with slightly less intensity [1-4]. Regarding normal tissues, STEAP1 expression is almost restricted to prostatic cells preferentially located on the plasma membrane of epithelial cells, particularly on cell-cell junctions, and to a lesser extend dispersed on the cytoplasm $[2,3,5]$. Although its precise cellular function is still not fully understood, STEAP1 appears to function as a transporter or ion channel, taking part on inter- and intracellular communication, possibly regulating cell proliferation and invasiveness $[1-3,6]$. Several studies have been pointed out the STEAP1 as a potential immunotherapeutic target as well as a biomarker, emphasizing its clinical relevance [7-11]. Besides STEAP1 gene, another related gene, STEAP1B, is encoded by the human genome. This gene is located at a different arm of the same chromosome as STEAP1, and may originate two different transcripts, STEAP1B1 and STEAP1B2. However, its expression in prostate cells is unstudied. Recently, our research group has demonstrated that STEAP1 is regulated by androgens and estrogens on the LNCaP cell line [5]. However, the mechanisms underlying STEAP1 over-expression on prostate cancer remain to be elucidated. It is well known that the regulation of gene expression could occur not only at transcription level but also at post-transcriptional and post-translational levels. In fact, events like cell-cycle progression, signal transduction and apoptosis, which are closely associated with oncogenesis, are influenced by mRNA stability as well as the rate of protein degradation [12-17]. It has been reported that tumors may activate endogenous mechanisms to increase mRNA stability of genes encoding oncogenes, cytokines, growth factors, and enzymes, leading to an 
enhanced protein over-expression, consequently raising cell growth and inflammatory processes known to be involved in the onset and progression of carcinogenesis [18-23]. Post-translational modifications (PTM) are intrinsically involved on regulating protein function, and therefore, are crucial for a variety of cellular processes, such as transcription, replication, cell cycle, apoptosis and cell signaling [24, 25]. Overall, we aimed to conduct an extensive in silico analysis of STEAP1 and STEAP1B, and to evaluate STEAP1 and STEAP1B expression in human prostate cell lines. In addition, the putative post-transcriptional and PTM modifications are evaluated through STEAP1 mRNA and protein stability, supplemented by a post-translational in silico analysis.

\section{RESULTS}

\section{STEAP1 and STEAP1B gene share high homology and are differentially expressed in human prostate cell lines}

A detailed in silico analysis allowed to compare the genomic organization of STEAP1 and STEAP1B genes. STEAP1 gene is found close to the telomeric region on chromosome $7 \mathrm{q} 21.13$, encoding a transcript with $1.3 \mathrm{~Kb}$, which originates a 339 aa mature protein (39.72KDa), with six predicted transmembranar regions, connected by three extracellular and two intracellular loops, and both $\mathrm{COOH}$ and $\mathrm{NH}_{2}$ intracellular terminal. The transmembrane domains are thought to be located between 73-95, 117-139, 164-182, 218-240, 252-274, 289-311 of the aa sequence (www.ncbi.nlm.nih.gov/ protein/NP_036581.1; http://www.cbs.dtu.dk/services/ TMHMM/) . STEAP1B gene is localized on chromosome 7 p15.3 and may originate two different transcripts, namely STEAP1B1 and STEAP1B2 (Figure 1). In comparison to the STEAP1 gene, STEAP1B1 has an additional exon, and a very large intron 4, with $53809 \mathrm{bp}$ on STEAP1B1 and $72728 \mathrm{bp}$ on STEAP1B2. STEAP1B1 is the longer transcript spanning approximately $1.3 \mathrm{~Kb}$, and may encode the longer isoform with 342 aa (39.547KDa), containing four potential transmembranar regions between aa 117-139, 163-182, 218-240 and 250-267, two intracellular and two extracellular loops, and $\mathrm{COOH}$ and $\mathrm{NH}_{2}$ intracellular terminal regions (www.ncbi.nlm. nih.gov/protein/NP_001157932.1; http:/www.cbs. dtu.dk/services/TMHMM/). STEAP1B2 transcript has approximately $1.2 \mathrm{~Kb}$, and may encode a protein with 245 aa $(28.684 \mathrm{KDa})$ with three potential transmembranar regions situated between aa 98-120, 144-163 and 199221 , one intracellular and one extracellular loops, an intracellular $\mathrm{NH}_{2}$ termini and an extracellular $\mathrm{COOH}$ region (www.ncbi.nlm.nih.gov/protein/NP_997225.1; http:/www.cbs.dtu.dk/services/TMHMM/) (Figure 1).
STEAP1B2 uses an alternate in-frame splice site in the 5 ' coding region and an alternate 3 ' exon with a distinct 3' coding region and 3' UTR, compared to variant 1 . The resulting isoform lacks an internal segment near the $\mathrm{N}$-terminus and has a shorter and distinct C-terminus when compared to isoform 1 (http://www.ncbi.nlm.nih.gov/ gene?term=STEAP1B). . This detailed analysis reveals that STEAP1 and STEAP1B1 isoforms share $89 \%$ and STEAP1 and STEAP1B2 91\% of homology (http://blast. ncbi.nlm.nih.gov/Blast.cgi).

STEAP1, STEAP1B1 and STEAP1B2 mRNA are differentially expressed in prostate cells lines. On the nonneoplastic prostate cells, PNT1A and PNT2, STEAP1, STEAP1B1 and STEAP1B2 mRNAs have little to no expression. On the other hand, on the malignant prostate cells, LNCaP and PC3, STEAP1 and STEAP1B2 are highly expressed, particularly STEAP1 (Figure 2A). STEAP1B1 mRNA is mainly expressed on PNT2 and PC3 cells, and under-expressed on LNCaP cells. The expression of STEAP1 protein was evaluated by western blot analysis (Figure 2B). Two immunoreactive bands could be identified, one of $30 \mathrm{KDa}$ on PNT1A and other of $36 \mathrm{KDa}$ on LNCaP cells. As seen in Figure 2B, STEAP1 protein is highly expressed on $\mathrm{LNCaP}$ cells, followed by PNT1A, PC3 and PNT2 with no expression.

\section{Stability of STEAP1 mRNA and protein in human prostate cell lines}

To evaluate the hypothesis that post-transcriptional and PTM mechanisms could be involved in the differential expression of STEAP1 between non-neoplastic and neoplastic cells, PNT1A and LNCaP cells were chosen to determine STEAP1 mRNA and protein stability through qPCR and western blot analysis, respectively. Cells were primarily cultured on CM, and STEAP1 mRNA stability was established by assessing the relative decay rates of STEAP1 after Act D treatment at 0, 1, 2, 4, 8 and 12h (Figure 3A). STEAP1 protein stability was determined after inhibiting protein synthesis with Chx for $0,1,2,4,6$, 8 and 16 h (Figure 3B). On PNT1A cells, the approximate half-life of STEAP1 mRNA levels is $4 \mathrm{~h}$, whereas on LNCaP is about $10 \mathrm{~h}$. Comparing the decaying mRNA levels of both cell lines, it is noticed that STEAP1 mRNA is more stable on LNCaP cells than on PNT1A cells. Regarding to STEAP1 protein expression after exposure to $\mathrm{Chx}$, the approximate half-life of STEAP1 protein on PNT1A cells is about 6h, whereas the levels on LNCaP are still high even after $16 \mathrm{~h}$ of treatment with Chx. In accordance to the mRNA data, it is clear that STEAP1 protein is also more stable on LNCaP than on PNT1A cells. However, no relationship seems to occur between the decay rates of mRNA and protein, namely on LNCaP cells.

It is well known that FBS (serum) contains hormones 

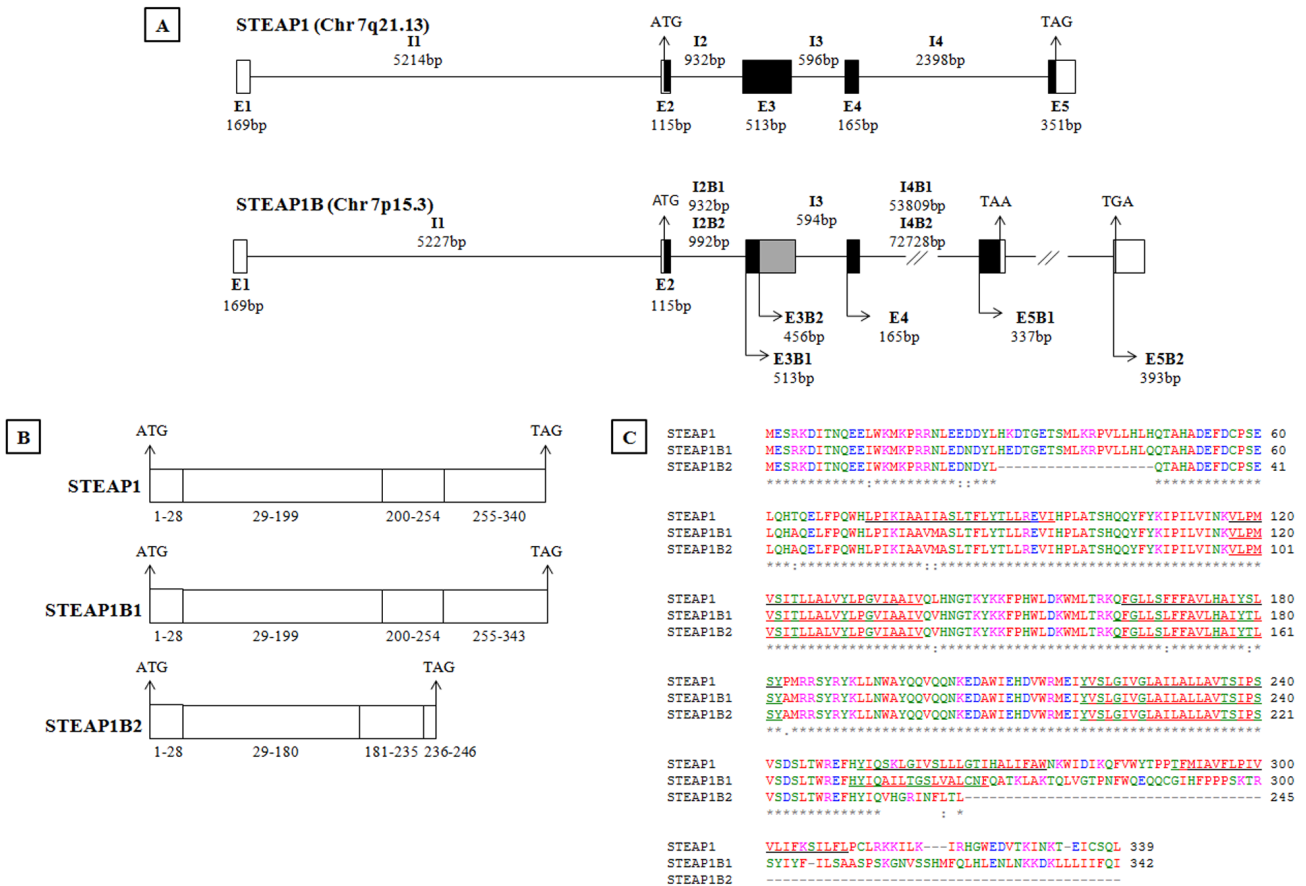

D
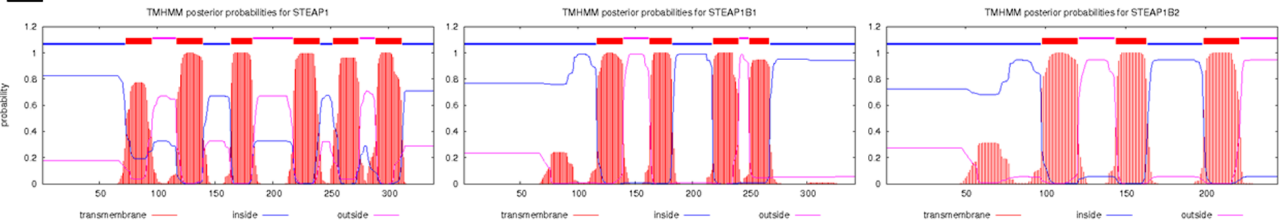

Figure 1: In silico analysis of human STEAP1 and STEAP1B gene. Genomic organization (A) and transcripts (B) resulting from STEAP1 and STEAP1B gene. Exons (E), Introns (I) and their molecular sizes in bp (base pairs) are indicated. The sequence ATG and TAG/ TAA corresponds to initiation and STOP codons, respectively. White boxes indicate non-coding exons, and black or grey boxes represent regions of coding exons depending on transcript encoded by STEAP1B gene. C- Alignment of amino acids sequences of STEAP1 and putative STEAP1B isoforms. The underlined amino acids sequences correspond to predicted transmembrane regions. * indicate identical amino acids among STEAP1s proteins; ":" indicate different amino acids but with similar physical-chemistry properties. D- Prediction of transmembrane helices in STEAP1, STEAP1B1 and STEAP1B2 proteins. All sequences were retrieved from http://genome.ucsc.edu/ and the alignment was carried out using Clustal Omega (https://www.ebi.ac.uk/Tools/msa/clustalo/). The prediction of transmembrane helices was performed resorting to Center for Biological Sequence analysis (http://www.cbs.dtu.dk/services/TMHMM/).
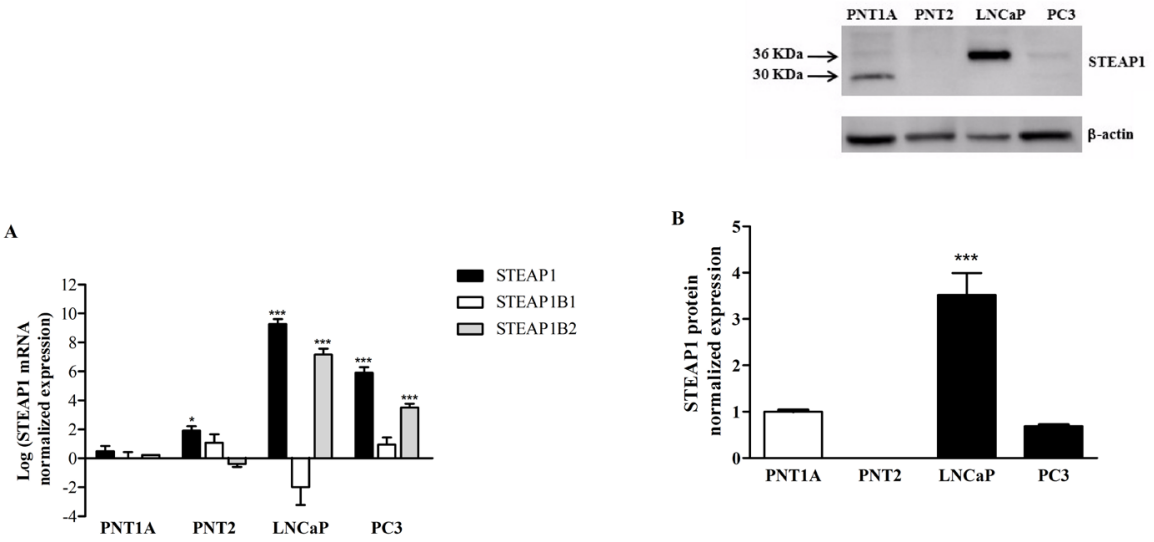

Figure 2: Differential expression of STEAP1, STEAP1B1 and STEAP1B2 on prostate cell lines. A. mRNA expression of STEAP1 and its isoforms were determined by qPCR. B. STEAP1 protein expression was determined by Western blot. mRNA and protein expression was normalized with hGAPDH and $\beta$-actin, respectively. Error bars indicate mean $\pm \mathrm{SEM}$ of $\mathrm{n}=6 .{ }^{*} \mathrm{p}<0.05,{ }^{* * *} \mathrm{p}<0.001$ (Oneway ANOVA followed by Bonferroni test) compared with PNT1A expression. 
and growth factors important to cell homeostasis. Therefore, the effect of serum on STEAP1 mRNA and protein stability was investigated. In the presence of FBS, STEAP1 mRNA expression increased 2 fold after $8 \mathrm{~h}$ of stimuli on PNT1A cells (Figure 4A, upper left), whereas on LNCaP cells STEAP1 mRNA expression decreased almost 2 fold after $12 \mathrm{~h}$ of stimuli with FBS (Figure 4B, lower left). The effect of FBS in STEAP1 protein levels at these time points was in accordance to STEAP1 mRNA, i.e., increased at $8 \mathrm{~h}$ on PNT1A (Figure 4A right upper) and decrease after $12 \mathrm{~h}$ on LNCaP cells (Figure 4B, lower right). These results show a clear opposite response to hormones and growth factors, which are present in FBS, in non-neoplastic and neoplastic prostate cell lines.

\section{In silico analysis of post-translational modifications in STEAP1 protein}

In an attempt to uncover the causes behind the above mentioned differences on STEAP1 protein stability, a closer insight into PTM was achieved through an extensive in silico analysis. Alterations such as N-glycosylation, glycation, phosphorylation sites and O- $\beta$-GlcNAc anchor sites on STEAP1 aa sequences were investigated. As seen on Figure 5A, two N-X-S/T consensus sequences [32] were found on STEAP1 sequence, but a single potential $\mathrm{N}$-glycosylation site was identified at position 143, corresponding to the asparagine aa. Although another asparagine was signaled, the potential score was below the threshold line. Potential glycation sites occur in several lysines (9/24) of STEAP1 protein. These potentially glycated aa are located at positions $5,15,17,30,108$, 148, 149, 156 and 162 (Figure 5B). It is well known that three distinct aa (serine, threonine and tyrosine) have the ability to be phosphorylated. According to the results retrieved from NetPhos 2.0 Server, four serines (positions 3, 187, 240 and 244), two threonines (positions 160 and 246) and four tyrosines (positions 27, 147, 219 and 252) are potentially phosphorylated on STEAP1 (Figure 5C). Several kinase enzymes with the potential to

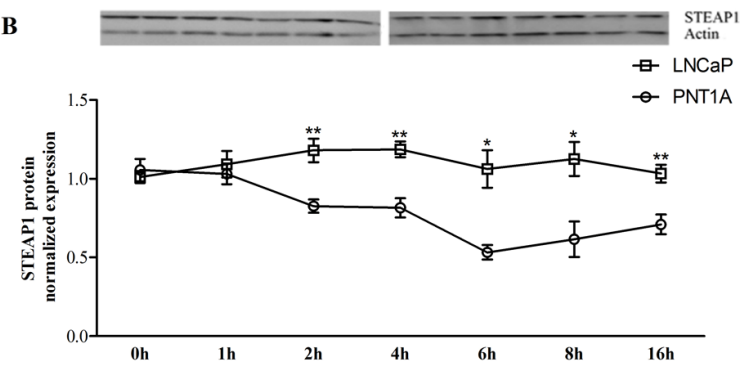

Figure 3: STEAP1 mRNA and protein stability in human prostate cell lines. Comparison between PNT1A and LNCaP STEAP1 mRNA (A) and protein (B) stability in complete culture medium. mRNA and protein expressions were determined by qPCR and Western blot, respectively. mRNA and protein expression was normalized with hGAPDH and $\beta$-actin, respectively. Error bars indicate mean \pm SEM of $n=6 .{ }^{*}<0.05, p^{* *}<0.01$ (t-test) comparing PNT1A and LNCaP STEAP1 expression at each indicated time.

A

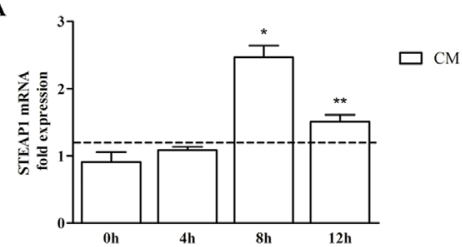

B

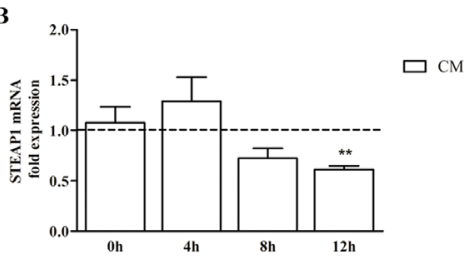

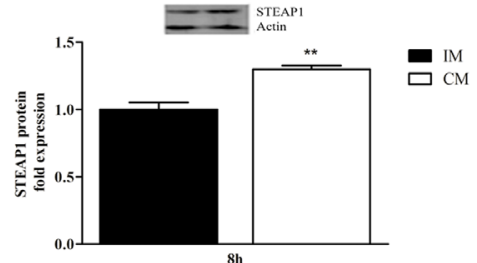

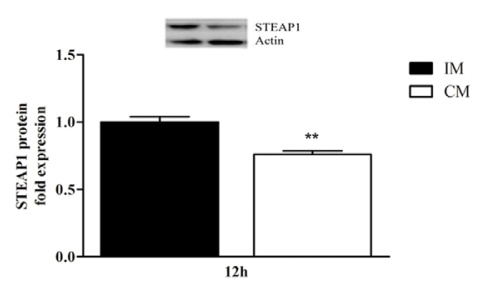

Figure 4: Effect of serum on PNT1A (A- upper left and right) and LNCaP (B- lower left and right) STEAP1 mRNA and protein stability, after treatment with Act D and Chx, respectively. mRNA and protein fold variation relatively to incomplete medium (IM) were determined by qPCR and Western blot, respectively. mRNA and protein expression was normalized with hGAPDH and with $\beta$-actin, respectively. Error bars indicate mean $\pm \mathrm{SEM}$ of $\mathrm{n}=6 .{ }^{*} \mathrm{p}<0.05,{ }^{*} \mathrm{p}<0.01$ (t- test) comparing (IM) and complete medium (CM) STEAP1 expression at each indicated time. 
Table 1: Sequences, amplicons sizes and annealing temperatures for the different STEAP1 mRNA and the internal controls used for quantitative Real-Time PCR.

\begin{tabular}{|c|c|c|c|}
\hline Oligo name & Sequence $\left(5^{\prime}-3^{\prime}\right)$ & Amplicon Size (bp) & $\begin{array}{l}\text { Anneling } \\
\text { temperature }\end{array}$ \\
\hline hSTEAP1_619fw & GGC GAT CCT ACA GAT ACA AGT TGC & \multirow{2}{*}{128} & \multirow{8}{*}{$60^{\circ} \mathrm{C}$} \\
\hline hSTEAP1_747rv & CCA ATC CCA CAA TTC CCA GAG AC & & \\
\hline hSTEAP1B1fw & CTGGAAGCCTGGTAGCTTTG & \multirow{2}{*}{162} & \\
\hline hSTEAP1B1rv & GGCTGGCTGCTGATAAAATG & & \\
\hline hSTEAP1B2fw & CGATTATTTGCAAACAGCCC & \multirow{2}{*}{173} & \\
\hline hSTEAP1B2rv & GGGAAGTTGCTAAAGGGTGA & & \\
\hline hGAPDH_74fw & CGC CCG CAG CCG ACA CAT C & \multirow{2}{*}{75} & \\
\hline hGAPDH_149rv & CGC CCA ATA CAA TCC G & & \\
\hline
\end{tabular}

phosphorylate different aa on STEAP1 sequence were also identified. As seen on Table 2, the threonine at position 160 presents the highest score to be phosphorylated by protein kinase C. GlcNAcylation usually occurs in parallel with phosphorylation, and the two are frequently mutually exclusive $[33,34]$. In fact, the potential O- $\beta$-GlcNAc anchor sites can be found on threonines 236 and 333, as well as on serines 237 and 242 of the STEAP1 aa sequence (Figure 5D).

\section{DISCUSSION}

STEAP1 is mainly expressed in normal and malignant prostate and the pursuit for understanding how its expression is regulated and modulated by internal and external factors is fundamental. Recently, Grunewald and colleagues described the existence of a STEAP1 homolog gene, named STEAP1B [35]. STEAP1 and STEAP1B genes are located on chromosome 7, but on different arms, i.e., STEAP1 gene is located at the long arm (7q21.13) and STEAP1B on the short arm (7p15.3). Considering the high homology between STEAP1 and STEAP1B genes, it is possible that gene duplication may have occurred during genome evolution. STEAP1B gene gives rise to two transcripts, STEAP1B1 and STEAP1B2, which share several similarities to STEAP1, but may encode two different mature proteins of 332 aa and 245 aa, respectively. Although the expression of STEAP1B at protein level still needs to be verified, these putative proteins also display transmembrane regions like STEAP1, but in a smaller number, suggesting that different roles could be played by these isoforms in comparison to STEAP1 protein. There are some common features present on the structure of STEAP family of proteins, such as the presence of an N-terminal with homology to the archaeal and bacterial $\mathrm{F}_{420} \mathrm{H}_{2}: \mathrm{NADP}^{+}$oxidoreductase (FNO)binding proteins and human NADPH-oxidoreductases, two conserved histidines that bind an intramembranar heme group known as the ACRATA domain, the presence of the Rossman fold within the N-terminal and the strikingly similarity between the C-terminal domain to the yeast FRE cytochrome $b$ metalloreductase domain, indicating their possible role as oxidoreductases [36-39]. Both STEAP1B isoforms, lack the FNO-like domain, the Rossman fold and the NADPH-oxidoreductase domain, as STEAP1 $[35,36]$. In addition, STEAP1B2 appears to be
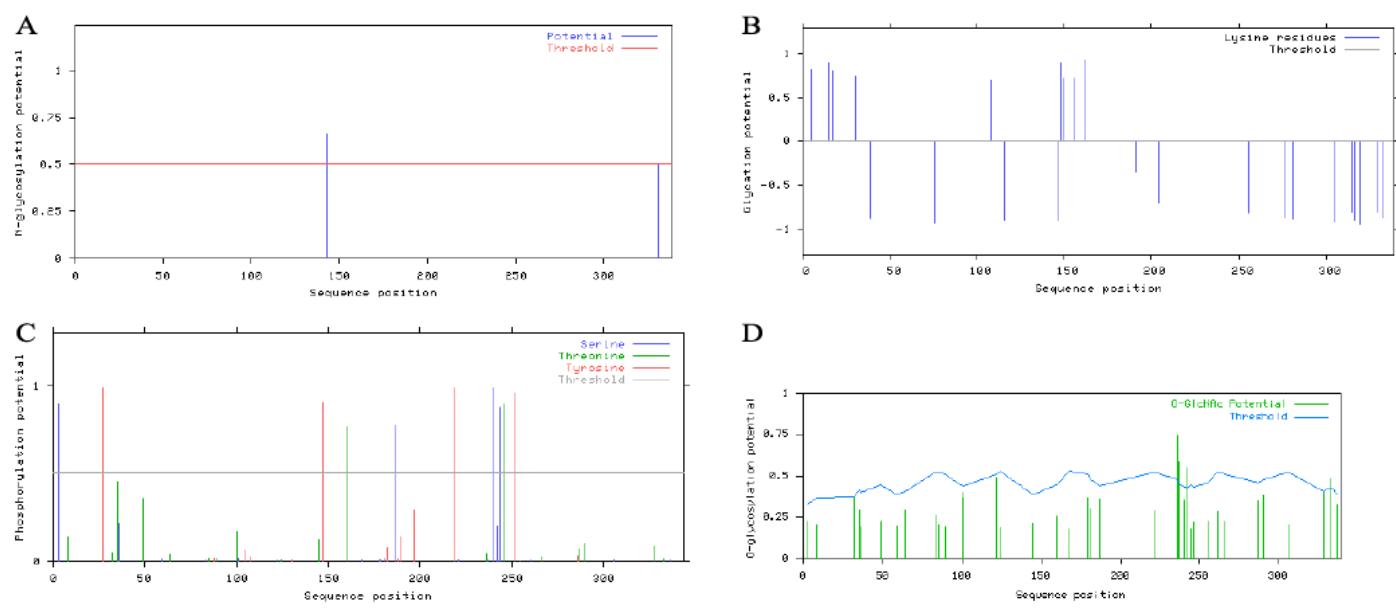

$\mathrm{D}$

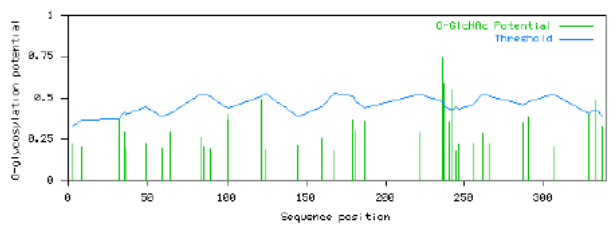

Figure 5: Prediction of N-glycosylation, glycation, phosphorylation and O- $\beta$-GlcNAc sites of STEAP1 using: A. NetNGlyc 1.0, B- NetGlycate 1.0, C. NetPhos 2.0 and D- YinOyang 1.2, respectively. 
Table 2: Putative Kinases enzymes involved on STEAP1 phosphorylation. Prediction was performed using NetPhosK 1.0 server.

\begin{tabular}{|l|l|l|}
\hline Site & Kinase & Score \\
\hline S-3 & PKC & 0,67 \\
\hline T-8 & CKII & 0,57 \\
\hline Y-27 & EGFR & 0,58 \\
\hline Y-27 & INSR & 0,55 \\
\hline T-64 & DNAPK & 0,59 \\
\hline T-64 & ATM & 0,60 \\
\hline S-83 & DNAPK & 0,55 \\
\hline S-83 & PKA & 0,74 \\
\hline T-85 & PKC & 0,64 \\
\hline T-100 & PKC & 0,67 \\
\hline S-122 & PKA & 0,67 \\
\hline T-145 & PKC & 0,59 \\
\hline T-160 & PKC & 0,91 \\
\hline S-179 & PKC & 0,59 \\
\hline S-181 & PKC & 0,68 \\
\hline S-187 & PKC & 0,87 \\
\hline S-187 & PKA & 0,58 \\
\hline Y-197 & EGFR & 0,60 \\
\hline S-221 & PKA & 0,55 \\
\hline T-246 & CKII & 0,53 \\
\hline T-246 & PKC & 0,86 \\
\hline S-255 & PKC & 0,78 \\
\hline S-255 & Cdc2 & 0,56 \\
\hline S-261 & PKA & 0,68 \\
\hline T-287 & Cdk5 & 0,52 \\
\hline S-306 & PKA & 0,63 \\
\hline T-333 & PKC & 0,63 \\
\hline S-337 & DNAPK & 0,58 \\
\hline S-337 & ATM & 0,64 \\
\hline
\end{tabular}

also missing the heme-binding sites, making it unfit to act as a ferric oxidoreductase [35].

STEAP1 mRNA overexpression is well described on LNCaP and PC3 cells [2]. We demonstrated that STEAP1B1 and STEAP1B2 mRNAs are also expressed on prostate cell lines. Alike in STEAP1, STEAP1B2 mRNA is also overexpressed in neoplastic cells when compared to non-neoplastic cells, suggesting that also STEAP1B2 may be dysregulated in cancer and demonstrating its potential application as a biomarker. STEAP1B1 mRNA can only be found in diminished levels on PNT2 and PC3 cells, and little or no expression on the LNCaP and PNT1A cells. These results seem to suggest that STEAP1B1 mRNA is not differentially expressed between normal and prostate cancer. However, it is needed to carry out studies using human prostate cancer cases to evaluate the expression profile of STEAP1B1 and STEAP1B2, as well as to clarify their clinical significance.

A strong immunoreactive band of approximately
$36 \mathrm{KDa}$ was detected on LNCaP cells, which must correspond to STEAP1 protein, as described by others [1, $2,6]$. Interestingly, an immunoreactive band of $30 \mathrm{KDa}$ was obtained on PNT1A cells. The different molecular weight of STEAP1 between LNCaP and PNT1A may result from different PTM on protein, as suggested by our results and discussed below. It is also possible that the immunoreactive band of $30 \mathrm{KDa}$ may correspond to STEAP1B2, because this is the predicted molecular weight of STEAP1B2 isoform. Nonetheless, further analysis using specific antibodies against STEAP1B1 and STEAP1B2 are required to determine their expression.

Numerous studies have described that STEAP1 is overexpressed in several kinds of tumors, but the mechanisms underlying its overexpression remain to be clarified. With this work we also attempted to unveil if STEAP1 expression would be regulated by post-transcriptional and PTM. Considering that both mRNA and protein stability display an important role at cellular level, in regulation of gene induction/expression, proliferation, cell signaling and apoptosis [12-16, 40], the STEAP1 stability in non-neoplastic and neoplastic prostate cell lines was evaluated. mRNA half-life and turnover are dependent on development stage or environmental factors, such cytokines, hormones, among others, decreasing its rate of transcription $[40,41]$. The regulation of mRNA stability is intrinsically associated to the regulation of protein production [40]. The most stable mRNA and proteins are usually associated with vital processes such as translation, metabolic pathways and respiratory machinery [42]. According to the collected data, STEAP1 mRNA and protein are less stable in the PNT1A cells in comparison to the highly stable STEAP1 mRNA and protein on LNCaP cells. The enhancement of STEAP1 stability in LNCaP cells suggests that post-transcriptional and PTM may differ between non-neoplastic and neoplastic cells, contributing for STEAP1 overexpression in cancer cells. The evaluation of the effect of serum on STEAP1 stability shows that serum has opposite effects on STEAP1 stability, increasing it on PNT1A and decreasing on LNCaP cells. Recently, our research group demonstrated that treatment with serum or DHT down-regulates STEAP1 expression through androgen receptor (AR) [5]. Considering that DHT increases AR mRNA stability and consequently the levels of AR protein, it is liable to speculate that DHT present on serum may contribute to decreased STEAP1 stability [43]. However, other factors may be involved in stability of STEAP1.

The opposite effect of serum in STEAP1 stability on PNT1A cells reinforce that the mechanisms involved in regulation of STEAP1 may differ between non-neoplastic and neoplastic cells. Furthermore, the increased stability of both mRNA and protein on LNCaP cells is in accordance that stable mRNAs allow a wider translational timeframe for genes that are expressed at high levels [44].

There are several factors that could control mRNA 
and protein turnover on cells, and in an attempt to determine possible PTM's in STEAP1 protein, in silico analysis was carried out. Four different types of PTM's were found to be likely to occur in STEAP1 protein, namely $\mathrm{N}$-glycosylation, glycation, phosphorylation and O- $\beta-$ GlcNAcylation. STEAP1 protein is therefore potentially subjected to multisite modification, a phenomenon that modulates protein function by "loss-offunction" and/or "gain-of-function" mechanisms as a result of interaction between the different modifications [25]. It is well documented that these types of modifications tend to confer higher stability to proteins and are often implicated on the etiology and pathogenesis of several diseases, including cancer [25, 45-50]. Furthermore, certain PTM's may even be used as diagnostic targets and are known to enhance tumor cell proliferation and invasion, as in prostate cancer [51-53]. Further analysis is required to ascertain if these particular PTM's are occurring on cells, and if they occur, it is crucial to evaluate the role of these modifications in carcinogenesis.

In conclusion, STEAP1B transcripts have similar structural features to STEAP1, but may encode proteins with less transmembrane domains. STEAP1B2 transcript is also overexpressed on neoplastic prostate, making it worth to evaluate its potential as cancer biomarker. For the first time, we demonstrated that STEAP1 expression and regulation could also be under the control of several PTM. Clarifying the regulation of STEAP1 as well as the expression and function of STEAP1B on cells may open novel strategies for diagnosis and treatment of prostate cancer.

\section{MATERIAL AND METHODS}

\section{Cell culture and treatment}

Human prostate cell lines (PNT1A, PNT2, LNCaP and $\mathrm{PC} 3$ ) were purchased from the European Collection of Cell Cultures (ECACC, Salisbury, UK). All cell lines were grown in RPMI 1640 medium (Gibco, Paisley, Scotland) supplemented with 10\% FBS (Biochrom AG, Berlin, Germany) and 1\% penicillin/streptomycin (Invitrogen, NY, USA), in a humidified chamber at $37^{\circ} \mathrm{C}$ and a $5 \% \mathrm{CO}_{2}$ atmosphere. Determination of STEAP1, STEAP1B1 and STEAP1B2 expression was determined through the collection of cells from three different passages. Evaluation of mRNA and protein stability was achieved with treatment with inhibitors of transcription [actinomycin D (Act D)], and translation [cycloheximide $(\mathrm{Chx})]$, respectively. Two different approaches were carried out in order to evaluate STEAP1 mRNA and protein stability in cells: a) LNCaP and PNT1A cells were grown up to $60 \%$ confluence in Complete Medium [(CM) RPMI 1640, 10\% FBS and1\% penicillin/streptomycin].
This medium was then replaced by CM supplemented with Act D $1 \mu \mathrm{g} / \mathrm{mL}$, or Chx $1 \mu \mathrm{g} / \mathrm{mL}$. Cells were harvested after $0 \mathrm{~h}, 1 \mathrm{~h}, 2 \mathrm{~h}, 4 \mathrm{~h}, 8 \mathrm{~h}$ and $12 \mathrm{~h}$ to assess mRNA stability, and after $0 \mathrm{~h}, 1 \mathrm{~h}, 2 \mathrm{~h}, 4 \mathrm{~h}, 8 \mathrm{~h}$ and $16 \mathrm{~h}$ for protein stability; b) LNCaP and PNT1A cells were grown up to $60 \%$ in Incomplete Medium [(IM) without serum]. This medium was posteriorly supplanted by CM or IM supplemented with Act D $1 \mu \mathrm{g} / \mathrm{mL}$, or Chx $1 \mu \mathrm{g} / \mathrm{mL}$. Cells were harvested after $0 \mathrm{~h}, 4 \mathrm{~h}, 8 \mathrm{~h}$ and $12 \mathrm{~h}$ for mRNA stability, and after $8 \mathrm{~h}$ and $12 \mathrm{~h}$ for protein stability in PNT1A and LNCaP cells, respectively.

\section{Total RNA extraction and cDNA synthesis}

Total RNA from human prostate cell lines was obtained using TRI reagent (Ambion, UK) according to the manufacturer's instructions. Total RNA integrity and quantification were assessed by agarose gel electrophoresis and measuring 260 and $280 \eta \mathrm{m}$ absorvances on a nanospectrometer (Pharmacia Biotech, Ultraspec 3000). cDNA synthesis was carried out using NZY FirstStrand cDNA Synthesis Kit (Nzytech, Lisboa, Portugal) according to protocol.

\section{Total Protein Extraction}

PNT1A, PNT2, LNCaP and PC3 cells were lysed on an appropriated volume of RIPA buffer (150 mM NaCl, $1 \%$ Nonidet-P40 substitute, $0.5 \%$ Na-deoxycholate, $0.1 \%$ SDS, $50 \mathrm{mM}$ Tris, $1 \mathrm{mM}$ EDTA) supplemented with 1\% protease cocktail and 10\% PMSF. The total protein extract (supernatant) was obtained after centrifugation the cell lysate for $20 \mathrm{~min}$ at $12000 \mathrm{rpm}$ and $4^{\circ} \mathrm{C}$. Quantification of the total protein bulk was measured using the Bradford method (Biorad, CA, USA).

\section{Quantitative Real-Time PCR analysis}

Quantitative real-time PCR (qPCR) was used to determine the expression of STEAP1, STEAP1B1 and STEAP1B2 in prostate cell lines and to establish PNT1A and LNCaP half-life of STEAP1 mRNA. qPCR reactions were performed on IQ5 Multicolor qPCR Detection System (Bio-Rad, Hercules, USA) using Maxima $^{\mathrm{TM}}$ SYBR Green/Fluorescein qPCR Master Mix (Thermo Scientific, Vilnius, Lithuania). The efficiency of qPCR was determined for all designated primers (Table 1) with serial dilutions $(1: 1 ; 1: 10 ; 1: 100 ; 1: 1,000)$ of the cDNA. qPCR reactions were performed using $1 \mu \mathrm{l}$ of $\mathrm{cDNA}$ in a $20 \mu 1$ reaction containing $10 \mu 1$ SYBR Green and $300 \mathrm{nM}$ of specific primers. After an initial denaturation at $95{ }^{\circ} \mathrm{C}$ for $5 \mathrm{~min}, 35$ cycles were carried out as follows: denaturation at $95{ }^{\circ} \mathrm{C}$ for $30 \mathrm{~s}$, annealing temperature for $30 \mathrm{~s}$ and polymerization at $72{ }^{\circ} \mathrm{C}$ for $20 \mathrm{~s}$. The amplified PCR 
fragments were analyzed by melting curves: reactions were heated from 55 to $95^{\circ} \mathrm{C}$ with 10 s holds at each temperature $\left(0.05^{\circ} \mathrm{C} / \mathrm{s}\right)$. Fold differences were calculated following the mathematical model proposed by Pfaffl [26].

\section{Western blot}

Approximately $80 \mu \mathrm{g}$ of total protein from PNT1A, PNT2, PC3 and LNCaP cells were used to assess the STEAP1 protein expression on the four cell lines, and to determine protein stability. The protocol followed as previously been described by us [27]. Briefly, LNCaP and PNT1A protein extracts were resolved on $12 \%$ SDS-PAGE electrophoresis gel and then transferred into a PVDF membrane (GE Healthecare, UK). After blockage with 3\% casein solution, membranes were incubated with STEAP1 rabbit polyclonal antibody against humanSTEAP1 (H105) diluted 1:300 (Santa Cruz Biotechnology, Santa Cruz). Membranes were then incubated with an alkaline phosphatase conjugated goat polyclonal antibody against rabbit IgG (GE Healthcare, UK). Finally, STEAP1 immunoreactivity was visualized using Molecular Imager FX (Biorad, Hercules) after a brief incubation with ECF substract. STEAP1 and $\beta$-actin expression levels were quantified by densitometry using Quantity One Software (Biorad). $\beta$-actin was used to normalize STEAP1 expression.

\section{Bioinformatic analysis}

mRNA and protein sequences of STEAP1, STEAP1B1 and STEAP1B2 were retrieved from the National Center for Biotechnology Information (http:// www.ncbi.nlm.nih.gov/). . Alignment of amino acid (aa) sequences was performed using Clustal Omega program (https://www.ebi.ac.uk/Tools/msa/clustalo/). To determine protein homology, the online Basic Local Alignment Search Tool program (BLAST) (http://blast. ncbi.nlm.nih.gov/Blast.cgi) was used. Prediction of putative transmembranar domains was determined using TMHMM Server v. 2.0 (http://www.cbs.dtu.dk/services/ TMHMM). Prediction of amino acid post-translational alterations such as N-glycosylation, N-Glycation, Phosphorylation and Phosphokinase specific sites, and addition sites of O-linked $\beta$-N-acetylglucosamine (O- $\beta$ GlcNAc) were carried out using several tools available on Expasy ((http://www.expasy.org/proteomics/posttranslational_modification), NetNGlyc 1.0, NetGlycate 1.0 [28], NetPhos 2.0 [29], NetPhosK 1.0 [30] and YinOYang 1.2 [31] software's, respectively.

\section{Statistical analysis}

Data from all experiences are shown as mean \pm SEM of $n=6$. The statistical significance of STEAP1, STEAP1B1 and STEAP1B2 was assessed using Oneway ANOVA followed by Bonferroni test. mRNA and protein stability experiments statistical data was obtained comparing PNT1A and LNCaP STEAP1 expression at each indicated time, using t-test.

\section{FINANCIAL SUPPORT}

This work was partially supported by the COMPETE program (Pest-C/SAU/UI0709/2011) of Portuguese Foundation for Science and Technology (FCT).

\section{REFERENCES}

1. Grunewald TG, Diebold I, Esposito I, Plehm S, Hauer K, Thiel U, da Silva-Buttkus P, Neff F, Unland R, MullerTidow C, Zobywalski C, Lohrig K, Lewandrowski U, Sickmann A, Prazeres da Costa O, Gorlach A, et al. STEAP1 is associated with the invasive and oxidative stress phenotype of Ewing tumors. Mol Cancer Res. 2012; 10(1):52-65.

2. Hubert RS, Vivanco I, Chen E, Rastegar S, Leong K, Mitchell SC, Madraswala R, Zhou Y, Kuo J, Raitano AB, Jakobovits A, Saffran DC and Afar DEH. STEAP: a prostate-specific cell-surface antigen highly expressed in human prostate tumors. Proc Natl Acad Sci U S A. 1999; 96(25):14523-14528.

3. Maia CJB, Socorro S, Schmitt F and Santos CR. STEAP1 is over-expressed in breast cancer and down-regulated by 17beta-estradiol in MCF-7 cells and in the rat mammary gland. Endocrine. 2008; 34(1-3):108-116.

4. Yang D, Holt GE, Velders MP, Kwon ED and Kast WM. Murine six-transmembrane epithelial antigen of the prostate, prostate stem cell antigen, and prostate-specific membrane antigen: prostate-specific cell-surface antigens highly expressed in prostate cancer of transgenic adenocarcinoma mouse prostate mice. Cancer Res. 2001; 61(15):5857-5860.

5. Gomes IM, Santos CR, Socorro S and Maia CJ. Six transmembrane epithelial antigen of the prostate 1 is downregulated by sex hormones in prostate cells. The Prostate. 2012; 73(6):605-613.

6. Challita-Eid PM, Morrison K, Etessami S, An Z, Morrison KJ, Perez-Villar JJ, Raitano AB, Jia X-C, Gudas JM, Kanner SB and Jakobovits A. Monoclonal antibodies to sixtransmembrane epithelial antigen of the prostate- 1 inhibit intercellular communication in vitro and growth of human tumor xenografts in vivo. Cancer Res. 2007; 67(12):57985805.

7. Alves PMS, Faure O, Graff-Dubois S, Cornet S, Bolonakis I, Gross D-A, Miconnet I, Chouaib S, Fizazi K, Soria JC, 
Lemonnier FA and Kosmatopoulos K. STEAP, a prostate tumor antigen, is a target of human CD8+ T cells. Cancer Immunol Immunother. 2006; 55(12):1515-1523.

8. Azumi M, Kobayashi H, Aoki N, Sato K, Kimura S, Kakizaki $\mathrm{H}$ and Tateno M. Six-transmembrane epithelial antigen of the prostate as an immunotherapeutic target for renal cell and bladder cancer. J Urol. 2010; 183(5):20362044.

9. Garcia-Hernandez MDLL, Gray A, Hubby B and Kast WM. In vivo effects of vaccination with six-transmembrane epithelial antigen of the prostate: a candidate antigen for treating prostate cancer. Cancer Res. 2007; 67(3):13441351.

10. Grunewald T, Ranft A, Esposito I, da Silva-Buttkus P, Aichler M, Baumhoer D, Schaefer K, Ottaviano L, Poremba $\mathrm{C}$ and Jundt G. High STEAP1 expression is associated with improved outcome of Ewing's sarcoma patients. Ann Oncol. 2012; 23(8):2185-2190.

11. Valenti MT, Dalle Carbonare L, Donatelli L, Bertoldo F, Giovanazzi B, Caliari F and Lo Cascio V. STEAP mRNA detection in serum of patients with solid tumours. Cancer Lett. 2009; 273(1):122-126.

12. Kirkpatrick DS, Denison C and Gygi SP. Weighing in on ubiquitin: the expanding role of mass-spectrometry-based proteomics. Nature cell biology. 2005; 7(8):750-757.

13. Hershko A and Ciechanover A. The ubiquitin system. Annu Rev Biochem. 1998; 67:425-479.

14. King RW, Deshaies RJ, Peters JM and Kirschner MW. How proteolysis drives the cell cycle. Science. 1996; 274(5293):1652-1659.

15. Hao $\mathrm{S}$ and Baltimore D. The stability of mRNA influences the temporal order of the induction of genes encoding inflammatory molecules. Nature immunology. 2009; 10(3):281-288.

16. Friedel CC, Dolken L, Ruzsics Z, Koszinowski UH and Zimmer R. Conserved principles of mammalian transcriptional regulation revealed by RNA half-life. Nucleic Acids Res. 2009; 37(17):e115.

17. Hollams EM, Giles KM, Thomson AM and Leedman PJ. mRNA stability and the control of gene expression: implications for human disease. Neurochemical research. 2002; 27(10):957-980.

18. Aghib DF, Bishop JM, Ottolenghi S, Guerrasio A, Serra A and Saglio G. A 3' truncation of MYC caused by chromosomal translocation in a human T-cell leukemia increases mRNA stability. Oncogene. 1990; 5(5):707-711.

19. Hollis GF, Gazdar AF, Bertness V and Kirsch IR. Complex translocation disrupts c-myc regulation in a human plasma cell myeloma. Mol Cell Biol. 1988; 8(1):124-129.

20. Jones TR and Cole MD. Rapid cytoplasmic turnover of c-myc mRNA: requirement of the 3' untranslated sequences. Mol Cell Biol. 1987; 7(12):4513-4521.

21. Lasa M, Mahtani KR, Finch A, Brewer G, Saklatvala J and Clark AR. Regulation of cyclooxygenase 2 mRNA stability by the mitogen-activated protein kinase p38 signaling cascade. Mol Cell Biol. 2000; 20(12):4265-4274.

22. Ross HJ, Sato N, Ueyama Y and Koeffler HP. Cytokine messenger RNA stability is enhanced in tumor cells. Blood. 1991; 77(8):1787-1795.

23. Schuler GD and Cole MD. GM-CSF and oncogene mRNA stabilities are independently regulated in trans in a mouse monocytic tumor. Cell. 1988; 55(6):1115-1122.

24. Seet BT, Dikic I, Zhou MM and Pawson T. Reading protein modifications with interaction domains. Nat Rev Mol Cell Bio. 2006; 7(7):473-483.

25. Yang XJ. Multisite protein modification and intramolecular signaling. Oncogene. 2005; 24(10):1653-1662.

26. Pfaffl MW. A new mathematical model for relative quantification in real-time RT-PCR. Nucleic Acids Res. 2001; 29(9):e45.

27. Gomes IM, Santos CR, Socorro S and Maia CJ. Six transmembrane epithelial antigen of the prostate 1 is downregulated by sex hormones in prostate cells. Prostate. 2012.

28. Johansen MB, Kiemer L and Brunak S. Analysis and prediction of mammalian protein glycation. Glycobiology. 2006; 16(9):844-853.

29. Blom N, Gammeltoft S and Brunak S. Sequence and structure-based prediction of eukaryotic protein phosphorylation sites. J Mol Biol. 1999; 294(5):1351-1362.

30. Blom N, Sicheritz-Ponten T, Gupta R, Gammeltoft S and Brunak S. Prediction of post-translational glycosylation and phosphorylation of proteins from the amino acid sequence. Proteomics. 2004; 4(6):1633-1649.

31. Gupta R and Brunak S. Prediction of glycosylation across the human proteome and the correlation to protein function. Pacific Symposium on Biocomputing Pacific Symposium on Biocomputing. 2002:310-322.

32. Schwarz F and Aebi M. Mechanisms and principles of $\mathrm{N}$-linked protein glycosylation. Current opinion in structural biology. 2011; 21(5):576-582.

33. Wang Z, Pandey A and Hart GW. Dynamic interplay between O-linked $\mathrm{N}$-acetylglucosaminylation and glycogen synthase kinase-3-dependent phosphorylation. Molecular \& Cellular Proteomics. 2007; 6(8):1365-1379.

34. Wang Z, Gucek M and Hart GW. Cross-talk between GlcNAcylation and phosphorylation: site-specific phosphorylation dynamics in response to globally elevated O-GlcNAc. Proceedings of the National Academy of Sciences. 2008; 105(37):13793-13798.

35. Grunewald TG, Bach H, Cossarizza A and Matsumoto I. The STEAP protein family: versatile oxidoreductases and targets for cancer immunotherapy with overlapping and distinct cellular functions. Biol Cell. 2012; 104(11):641657.

36. Ohgami RS, Campagna DR, McDonald A and Fleming MD. The Steap proteins are metalloreductases. Blood. 2006; 108(4):1388-1394.

37. Finegold AA, Shatwell KP, Segal AW, Klausner RD 
and Dancis A. Intramembrane bis-heme motif for transmembrane electron transport conserved in a yeast iron reductase and the human NADPH oxidase. J Biol Chem. 1996; 271(49):31021-31024.

38. Ohgami RS, Campagna DR, Greer EL, Antiochos B, Mcdonald A, Chen J, Sharp JJ, Fujiwara Y, Barker JE and Fleming MD. Identification of a ferrireductase required for efficient transferrin-dependent iron uptake in erythroid cells. Nat Genet. 2005; 37(11):1264-1269.

39. Sanchez-Pulido L, Rojas AM, Valencia A, Martinez-A $\mathrm{C}$ and Andrade Ma. ACRATA: a novel electron transfer domain associated to apoptosis and cancer. BMC Cancer. 2004; 4:98.

40. Guhaniyogi J and Brewer G. Regulation of mRNA stability in mammalian cells. Gene. 2001; 265(1-2):11-23.

41. Ross J. mRNA stability in mammalian cells. Microbiological reviews. 1995; 59(3):423-450.

42. Wagner A. Energy constraints on the evolution of gene expression. Mol Biol Evol. 2005; 22(6):1365-1374.

43. Yeap BB, Krueger RG and Leedman PJ. Differential posttranscriptional regulation of androgen receptor gene expression by androgen in prostate and breast cancer cells. Endocrinology. 1999; 140(7):3282-3291.

44. Russell JE, Morales J and Liebhaber SA. The role of mRNA stability in the control of globin gene expression. Prog Nucleic Acid Re. 1997; 57:249-287.

45. Hanson SR, Culyba EK, Hsu TL, Wong CH, Kelly JW and Powers ET. The core trisaccharide of an N-linked glycoprotein intrinsically accelerates folding and enhances stability. Proc Natl Acad Sci U S A. 2009; 106(9):31313136.

46. Shental-Bechor D and Levy Y. Effect of glycosylation on protein folding: a close look at thermodynamic stabilization. Proc Natl Acad Sci U S A. 2008; 105(24):8256-8261.

47. Ling X, Sakashita N, Takeya M, Nagai R, Horiuchi S and Takahashi K. Immunohistochemical distribution and subcellular localization of three distinct specific molecular structures of advanced glycation end products in human tissues. Lab Invest. 1998; 78(12):1591-1606.

48. Yang WH, Kim JE, Nam HW, Ju JW, Kim HS, Kim YS and Cho JW. Modification of p53 with O-linked $\mathrm{N}$-acetylglucosamine regulates p53 activity and stability. Nature cell biology. 2006; 8(10):1074-1083.

49. Lynch TP, Ferrer CM, Jackson SR, Shahriari KS, Vosseller K and Reginato MJ. Critical role of O-Linked beta-N-acetylglucosamine transferase in prostate cancer invasion, angiogenesis, and metastasis. J Biol Chem. 2012; 287(14):11070-11081.

50. Chu C-S, Lo P-W, Yeh Y-H, Hsu P-H, Peng S-H, Teng Y-C, Kang M-L, Wong C-H and Juan L-J. O-GlcNAcylation regulates EZH2 protein stability and function. Proceedings of the National Academy of Sciences. 2014; 111(4):13551360.

51. Yoon SJ, Park SY, Pang PC, Gallagher J, Gottesman JE,
Dell A, Kim JH and Hakomori SI. N-glycosylation status of beta-haptoglobin in sera of patients with prostate cancer vs. benign prostate diseases. Int J Oncol. 2010; 36(1):193-203.

52. Fujimura T, Shinohara Y, Tissot B, Pang PC, Kurogochi M, Saito S, Arai Y, Sadilek M, Murayama K, Dell A, Nishimura S and Hakomori SI. Glycosylation status of haptoglobin in sera of patients with prostate cancer vs. benign prostate disease or normal subjects. Int $\mathrm{J}$ Cancer. 2008; 122(1):39-49.

53. Kamigaito T, Okaneya $\mathrm{T}$, Kawakubo M, Shimojo H, Nishizawa $\mathrm{O}$ and Nakayama J. Overexpression of O-GlcNAc by prostate cancer cells is significantly associated with poor prognosis of patients. Prostate Cancer Prostatic Dis. 2014; 17(1):18-22. 